\title{
Control of an Oscillatory Rectangular Cavity Jet Flow by Secondary Injection*
}

\author{
Mauro Parreira ARRUDA**, Nicholas John LAWSON** and Malcolm Roderik DAVIDSON***
}

\begin{abstract}
A method for controlling the position of an oscillatory cavity jet flow is demonstrated. The method involves secondary injection of a lower mass flow control jet into the cross-flow region of the primary jet. The primary jet in this case is a turbulent jet $(R e=55000)$ which when injected into the rectangular cavity with no secondary control, attains a stable oscillation with a characteristic Strouhal number of $S_{t, W}=0.013$. The injection control method is investigated using a combined experimental and numerical approach with a water model test rig and a 2D and 3D computational fluid dynamics (CFD) model. Based on previous work, a baseline cavity, with a depth to width ratio of $H / W=0.16$ and entry nozzle submergence of $S / W=0.38$, is used to study the effect of secondary jet injection parameters on primary jet deflection angle $(\delta)$ as a function of momentum ratio $(\beta)$ and injection position $\left(Y_{i}\right)$. Results have shown primary jet deflection angles $(\delta)$ of up to $15^{\circ}$ for a momentum ratio $(\beta)$ of $20 \%$ can be achieved for a secondary jet injection position of $Y_{1} / W=0.12$.
\end{abstract}

Key Words: Oscillatory Flow, Flow Control, Secondary Flow, LDV, CFD

\section{Introduction}

Flow control methods give the engineer the ability to manipulate a flow field to provide performance improvements and environmental benefits ${ }^{(1),(2)}$. Fluidic flow control involves flow field manipulation by either fluidic injection or zero flux pressure wave addition. These techniques offer advantages over mechanical methods including higher response rates and design simplicity with fewer moving parts. Fluidic flow control also allows control of frequency, amplitude and phase with good insensitivity to electromagnetic interference. The majority of published fluidic jet flow control studies have been for high Reynolds number flows ${ }^{(3)-(5)}$. However, there is little published on fluidic jet flow control at low Reynolds numbers which has potential application to MAV (Micro-Air-Vehicle) and UAV (Unmanned-Air-Vehicle) systems. Such systems may offer potential weight savings and possible efficiency benefits.

The following flow control technique is based on a primary turbulent jet injected into a rectangular cavity.

* Received 17th February, 2006 (No. 06-5032)

** Department of Aerospace Sciences, Cranfield University, Cranfield MK43 0AL U.K.

E-mail: n.lawson@cranfield.ac.uk

*** Department of Chemical and Biomolecular Engineering, The University of Melbourne, Parkville 3010, Australia
These type of flows have large scale self sustained oscillations with jet oscillation frequency primarily dependent on cavity width $^{(6)-(10)}$. In this case, the mechanism of oscillation is known to rely on the interaction between the two major recirculation zones formed within the main jet and the cavity sidewalls. In this system a feedback loop connects two recirculation zones and must be present for primary jet oscillation to occur. The jet oscillation frequency $f$ can be simply characterised by a Strouhal number $S_{t, l *}$ based on a cavity length scale $\left(l^{*}\right)$, usually the cavity width $(W)$ or the inlet nozzle diameter $\left(d_{1}\right)$ and the mean jet velocity $V$ such that:

$$
S_{t, l^{*}}=\frac{f \cdot l^{*}}{V}
$$

The following work demonstrates a fluidic flow control method by which the jet position is controlled through manipulation of the feedback loop by using secondary injection. This feedback loop manipulation determines the position of the recirculation zones and hence the primary jet angle. The performance of the system is assessed by using a combination of experimental and numerical modelling. The experimental approach is based on a physical water model with a primary jet Reynolds number of $R e=54000$ and laser Doppler anemometry (LDA) measurements. The numerical model uses commercial available code. Results outline the most important primary jet characteristics and their influence on the fluidic flow con- 
trol system. The results also provide recommendations for an optimum configuration including secondary jet injection characteristics and position.

\section{Nomenclature}

2D/3D : Two/Three-dimensional

CFD : Computational Fluids Dynamics

$d:$ Jet nozzle diameter

$f:$ Frequency of primary jet oscillation

$H$ : Depth of rectangular cavity (see Fig. 3)

$K s:$ Roughness height in wall function

LDA : Laser Doppler Anemometry

$\dot{m}$ : Mass flow rate

newf : Non-equilibrium wall functions

$R e:$ Reynolds number

$r m s$ : Root-mean-square value

$S:$ Submergence of primary jet nozzle (see Fig. 3)

$u, v, w:$ Flow velocity components

$V:$ Mean jet velocity

$W:$ Width of rectangular cavity (see Fig. 3)

$x, y, z:$ Cartesian axes system

$Y_{i}$ : Secondary jet position (see Fig. 3)

$Y_{R}$ : Position of jet re-attachment point on side wall (see Fig. 3)

$\alpha$ : Mass flow ratio between primary and secondary jets

$\beta$ : Momentum ratio between primary and secondary jets

$\delta:$ Primary jet deflection angle (see Fig. 3)

$\delta_{1,2}:$ Deflection angles (see Fig. 4)

\section{Subscripts}

$i, \mathrm{o}:$ Inner/Outer

1,2 : Primary/Secondary jet

\section{Numerical Modelling}

The 2D and 3D CFD transient models were developed using the commercial CFD solver (CFLUENT and mesh builder (C)GAMBIT. Figure 1 (a) and (b) illustrates the 3D mesh used for the modelling.

The 2D model was based on previous modelling methods ${ }^{(11)}$, whereby flow resistance in the primary jet nozzle region is modelled by a porous region with momentum losses proportional to the square of the local flow velocity. A number of turbulence models were tested on the baseline cavity configuration with no secondary injection. These models included the standard k- $\varepsilon$ model, the standard $\mathrm{k}-\varepsilon$ non-equilibrium wall functions (newf) model, the realizable $\mathrm{k}-\varepsilon$ model and the realizable $\mathrm{k}-\varepsilon$ newf model.

In terms of time-averaged and time-resolved characteristics, the standard k- $\varepsilon$ model gave the best overall predictions when compared to the LDA data. This model was then used for the remainder of the secondary injection modelling.

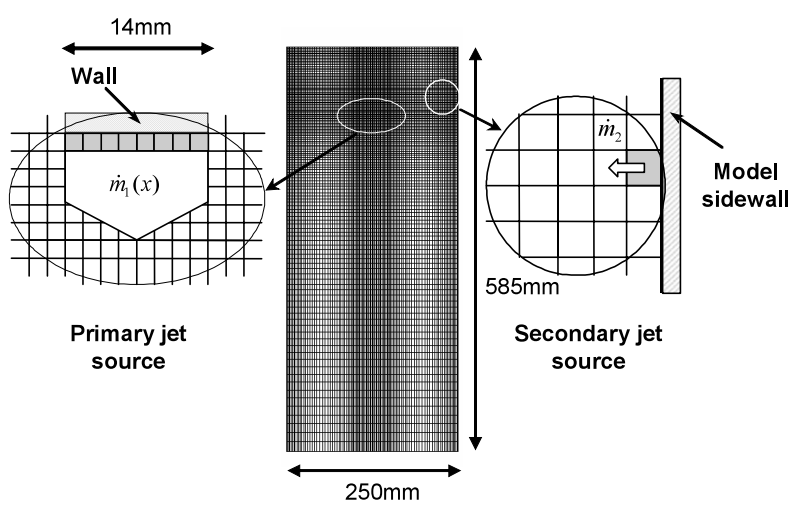

(a) 2D and 3D CFD model mesh configuration and source regions sketch $(2 \mathrm{D}-102 \times 130,3 \mathrm{D}-102 \times 130 \times 22)$

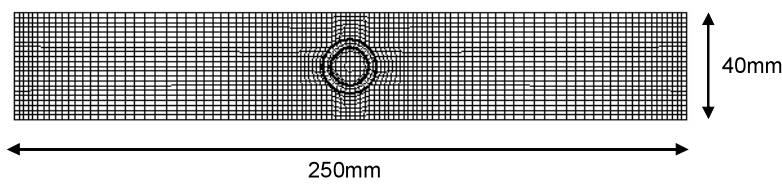

(b) 3D CFD $(102 \times 130 \times 22)$ model mesh configuration

Fig. $12 \mathrm{D}$ and 3D CFD model mesh configuration

Flow conditions in the near-wall region were also found to influence the natural mechanism of flow oscillation. An equivalent sand-grain roughness with uniform spherical distribution has been adopted for the near-wall flow treatment. Temporal characteristics of the flow were significantly influenced by increasing the wall roughness height $(K s)$ which resulted in higher shear stresses at the wall. Hence, velocity gradients in the near-wall region were accentuated, increasing local turbulence levels resulting in more rapid attachment of the flow to the sidewalls. Results showed that the 3D CFD solution became independent from the wall roughness height for values of $K s$ above $1 \mathrm{~mm}$ and therefore this value was used for the remainder of the calculations. Further numerical modelling details are given by Arruda ${ }^{(14)}$. Generally, the boundary layer characteristics in this region were observed to be highly unstable.

\section{Experimental Set-Up}

\section{1 Physical modelling}

Figure 2 shows the experimental set-up which consisted of a glass-walled rectangular cavity with dimensions $250 \mathrm{~mm} \times 585 \mathrm{~mm} \times 40 \mathrm{~mm}$ with a primary jet generated using a submerged glass-walled entry nozzle with diameters of $d_{i}=14 \mathrm{~mm}$ and $d_{o}=20 \mathrm{~mm}$ and an effective submergence of $S=95 \mathrm{~mm}$. The nozzle flow supply was provided by mounting a water tank above the cavity to isolate the unsteadiness of the pumps. A gate valve was used to control the inlet nozzle flow rate and the header tank was supplied from a reservoir below the cavity through $45 \mathrm{~W}$ and $250 \mathrm{~W}$ centrifugal water pumps. A slot valve was also mounted onto the cavity exit to ensure even outflow. Flow meters were mounted on both the primary and 


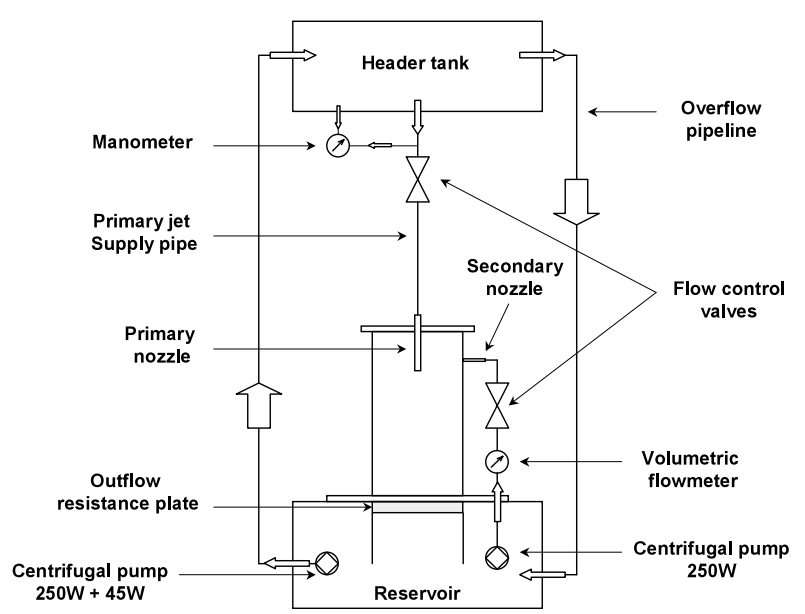

Fig. 2 Cavity configuration and experimental apparatus

secondary jet inputs. Three secondary jet nozzle sizes of diameters $5 \mathrm{~mm}, 6 \mathrm{~mm}$ and $7 \mathrm{~mm}$ were chosen to give the required range of mass flow and momentum for all the tests. This was confirmed though mass flow measurements through the nozzles.

\subsection{Flow measurement}

The primary flow measurement system used, to record flow velocity and turbulence inside the cavity, was a Dantec FibreFlow 2D laser Doppler anemometry (LDA) system. The system consisted of Dantec Fibre Flow optics, a backscatter probe and two BSA Enhanced processor boxes. The green $(514.5 \mathrm{~nm})$ and blue $(488 \mathrm{~nm})$ lines provided the two velocity components and the flow was seeded with glass Ballotini micro-spheres of diameters within a $30-50 \mu \mathrm{m}$ range. The probe had a focal length of $310 \mathrm{~mm}$ corresponding to a measurement volume of $0.047 \mathrm{~mm} \times 0.047 \mathrm{~mm} \times 0.381 \mathrm{~mm}$. Velocity bursts were set to a resolution of 32 with a maximum acquisition period of $30 \mathrm{~s}$. Average data rates were around $1000 \mathrm{~Hz}$ and ensured adequate temporal resolution for the oscillatory characteristics of the primary jet. The $u$ and $v$ LDA velocity data were subsequently processed into mean and rms $u_{r m s}, v_{r m s}$ values as outlined previously ${ }^{(12)}$. Data was recorded on a plane positioned about the cavity centreline and cross-flow region. Expected errors in the LDA velocity measurement $\left(\delta V_{\mathrm{LDA}} / V_{\mathrm{LDA}}\right)$ range between $4.2 \%-9.6 \%$ and $4.3 \%-9.7 \%$ for the primary and secondary jet flow meters $\left(\delta V_{1 / 2} / V_{1 / 2}\right)$ as calculated by Arruda $^{(13),(14)}$. Resultant errors from these measurements are the mass flow ratio $(\delta \alpha / \alpha)=6.0 \%$ and momentum ratio $(\delta \beta / \beta)=12.1 \%$.

Major variables used for the analysis are outlined in Figs. 3 and 4. The main variables used to assess flow control performance are the jet deflection angle $\delta$ and reattachment point $Y_{R}$ for a given momentum ratio $\beta$ and secondary jet position $Y / W$.

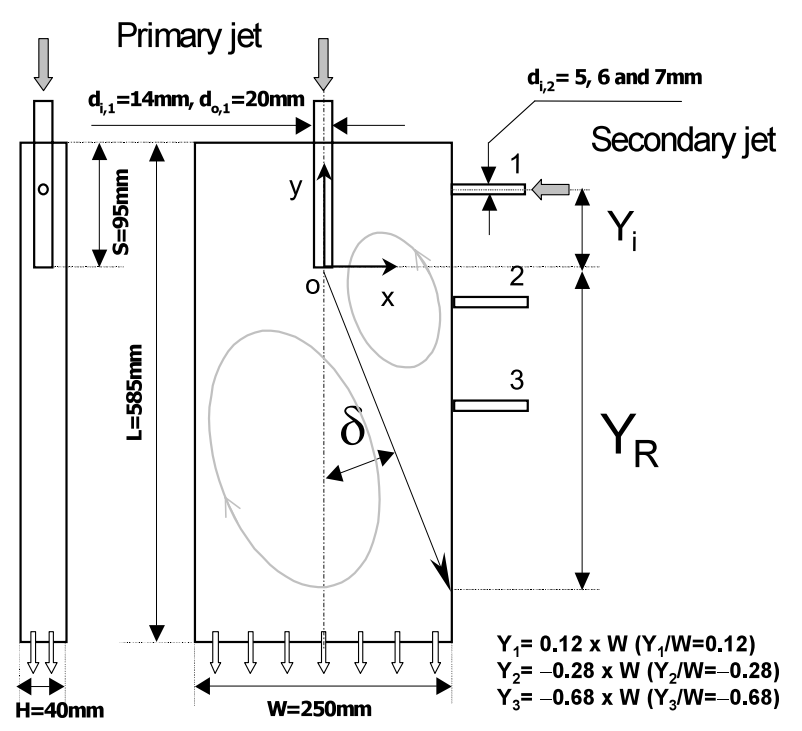

Fig. 3 Major variables for cavity flow analysis

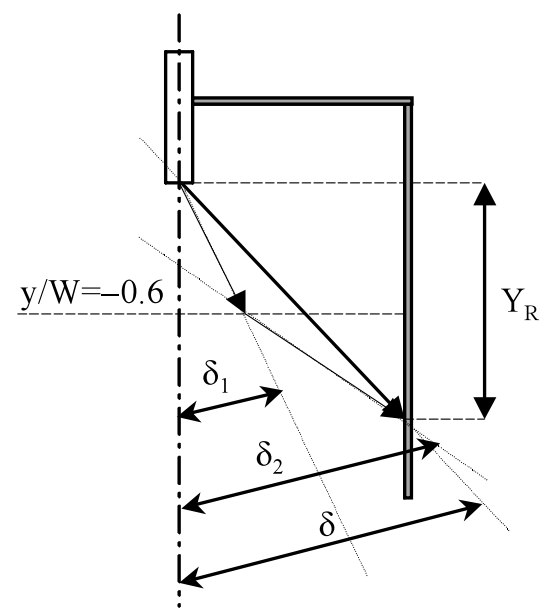

Fig. 4 Sketch of computed angles

$$
\begin{aligned}
& \beta=\frac{d_{i, 2}^{2} \cdot V_{2}^{2}}{d_{i, 1}^{2} \cdot V_{1}^{2}} \\
& \frac{Y_{R}}{W}=\frac{0.5-0.6 \tan \left(\delta_{1}\right)}{\arctan \left(\delta_{2}\right)}+0.6 \\
& \delta=\arctan \left(\frac{0.5}{Y_{R} / W}\right)
\end{aligned}
$$

Two angles $\delta_{1}$ and $\delta_{2}$ are taken from the centroid of the velocity profile at a fixed reference point $y / W=-0.6$ These are used to define the mean jet flow centreline reattachment point $\left(Y_{R}\right)$ on the sidewall and correspondent deflection angle $(\delta)$. This approach allows the prediction of mean reattachment points tending to infinity corresponding to a zero mean flow deflection angle. More specifically the angles $\delta_{1}$ and $\delta_{2}$ are formed from the nozzle exit centre, the centroid of the velocity profile and the mean deflection angle of the velocity profile vectors at $y / W=-0.6$. The point $y / W=-0.6$ was chosen as a clear inflection of the jet curvature was observed at this point during initial tests of the uncontrolled oscillatory jet. These angles are illus- 
trated in Fig. 4 and defined in the equations below to allow relative changes of the primary jet position to be assessed in comparison to an uncontrolled jet where $\delta=0$.

$$
\begin{aligned}
& \delta_{1}=\arctan \left[\frac{1}{2 Y_{p}} \cdot \frac{\sum_{i=2}^{N}\left[\left(X_{i}^{2}-X_{i-1}^{2}\right)\left(v_{i}+v_{i-1}\right)\right]}{\sum_{i=2}^{N}\left[\left(X_{i}-X_{i-1}\right)\left(v_{i}+v_{i-1}\right)\right]}\right] \\
& \delta_{2}=\frac{1}{N} \sum_{i=1}^{N} \arctan \left(\frac{u_{i}}{v_{i}}\right)
\end{aligned}
$$

Here $Y_{p}$ is the downstream position of the horizontal line of measured points $\left(Y_{p}=0.6 \mathrm{~W}\right), X_{i}$ the horizontal position of the measured point and $u$ and $v$ are the velocity components in the cross-stream and downstream directions. $N$ is the number of measured points $(N=19)$.

\section{Results and Discussion}

\subsection{LDA results}

Figure 5 shows the jet deflection angle $\delta$ obtained from the LDA data for a given range of secondary jet positions $Y / W$ and momentum ratios $\beta$. Results showed that when secondary injection is above the nozzle the primary jet deflection is to the same side as the injection. When secondary injection is below the nozzle, however, the primary jet deflection is to the opposite side. Hence there are two different methods of primary jet flow control where the first is by feedback loop control when injecting into the cross-flow region above the nozzle exit and the second is by forced deflection when injecting below the nozzle exit. In this case the feedback loop involves connection of the cross flow region above the nozzle exit through the two primary jet recirculation zones ${ }^{(8)}$. The results indicate that the greatest jet deflection sensitivity occurs at a secondary injection position of $Y_{1} / W=0.12$, i.e. when injecting into the cross-flow region. This result can be attributed to a jet entrainment mechanism which acts on a small recirculation region adjacent to the secondary jet. This low pressure region provides a net force which promotes crossflow and corresponding jet deflection. A similar analogy involves the secondary jet reinforcing on the same side of the jet with a resultant change in the primary jet position. Injecting below the nozzle relies on a mechanism of secondary jet momentum transfer to deflect the primary jet and given the high momentum of the primary jet, this method is relatively ineffective. Thus all further analysis

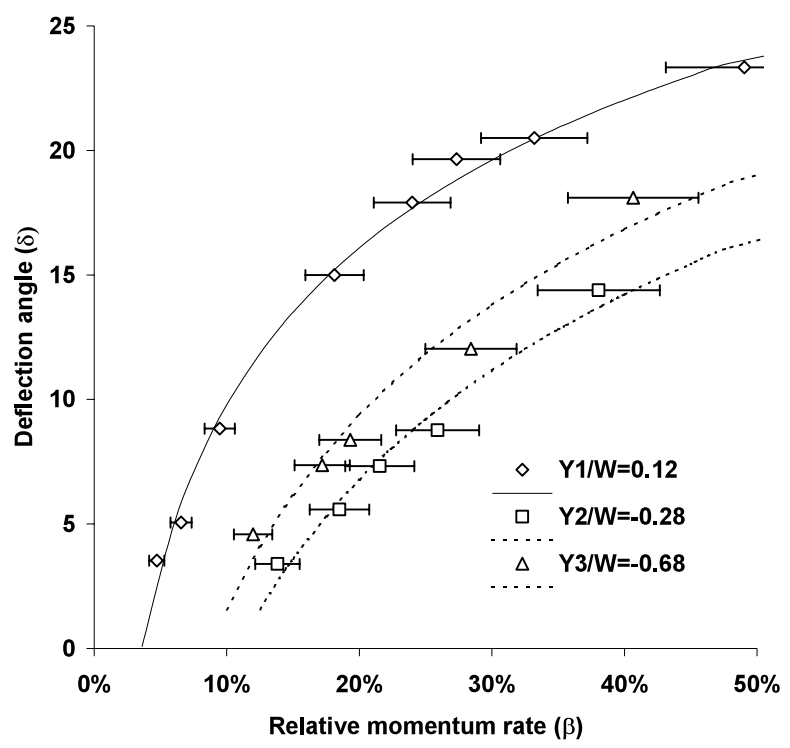

Fig. 5 LDA results showing jet deflection characteristics

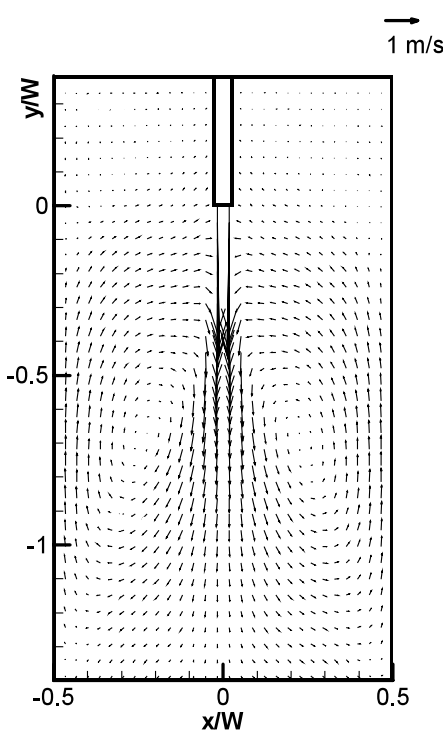

(a)

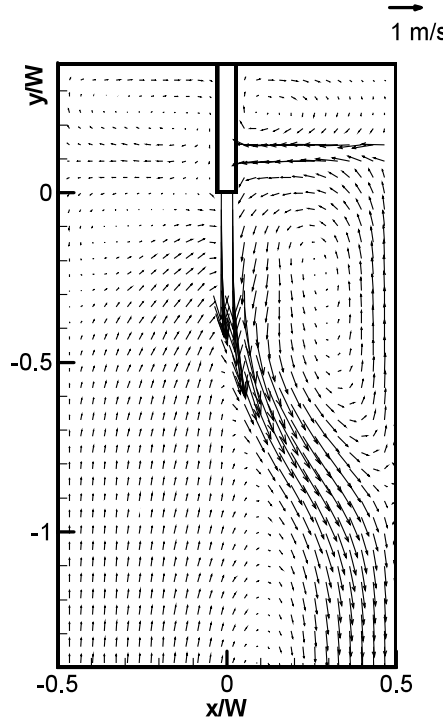

(b)

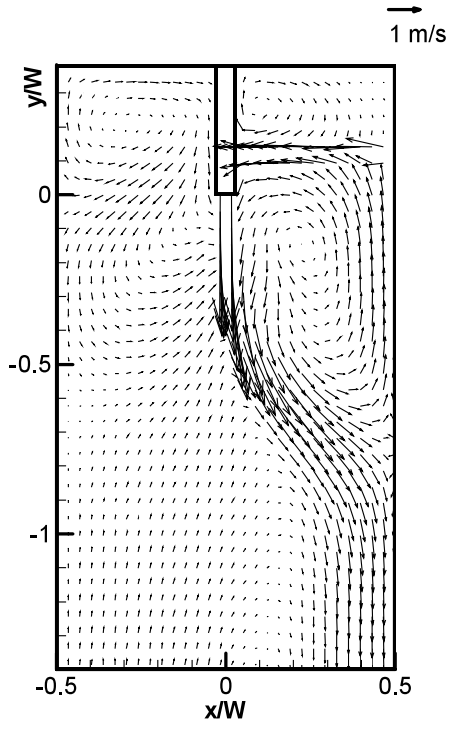

(c)

Fig. 6 3D CFD Characteristics for side injection (Contours of $V / V_{1}$ with $V_{1}=3.75 \mathrm{~m} / \mathrm{s}$, $Y_{1} / W=0.12$ ) (a) $\beta=0$ (b) $\beta=24 \%$ (c) $\beta=48.2 \%$ 
was restricted to secondary injection above the nozzle.

\subsection{CFD results}

Initial comparisons of the 2D CFD model with secondary injection with the LDA gave unacceptable predictions due to the inability of the 2D model to accurately capture the real flow structures in the region of injection. In contrast, the 3D CFD model accurately captured the jet deflection characteristics. Centreline results from the 3D CFD are shown in Fig. 6 where the jet deflection can be clearly seen. These comparisons between the $2 \mathrm{D}$ and 3D CFD are also shown in Fig. 7 in terms of jet deflection $\delta$ for a range of injection conditions of $\beta$. Here the empirical model is represented by a best fit curve of the experimental data. Overall, the results showed the key to control of primary jet position is the momentum ratio $\beta$ providing the secondary injection is positioned above the primary jet nozzle exit as found in the experimental results. In this configuration, primary jet deflection angles as high as $23.3^{\circ}$ for $24.6 \%$ of relative secondary mass flow momentum $\beta$ have been achieved.

\subsection{Temporal results}

Figure 8 shows the time history and spectral characteristics of the primary jet from LDA and 3D CFD data for a secondary injection momentum of $\beta=0$ and $\beta=49 \%$. The characteristics are generated from a baseline moni- toring point in the cross-flow region $(x=0, y / W=-0.6$, $z=0$ ).

Without secondary injection $(\beta=0)$, the jet was found to oscillate as expected with a dominant frequency cor-

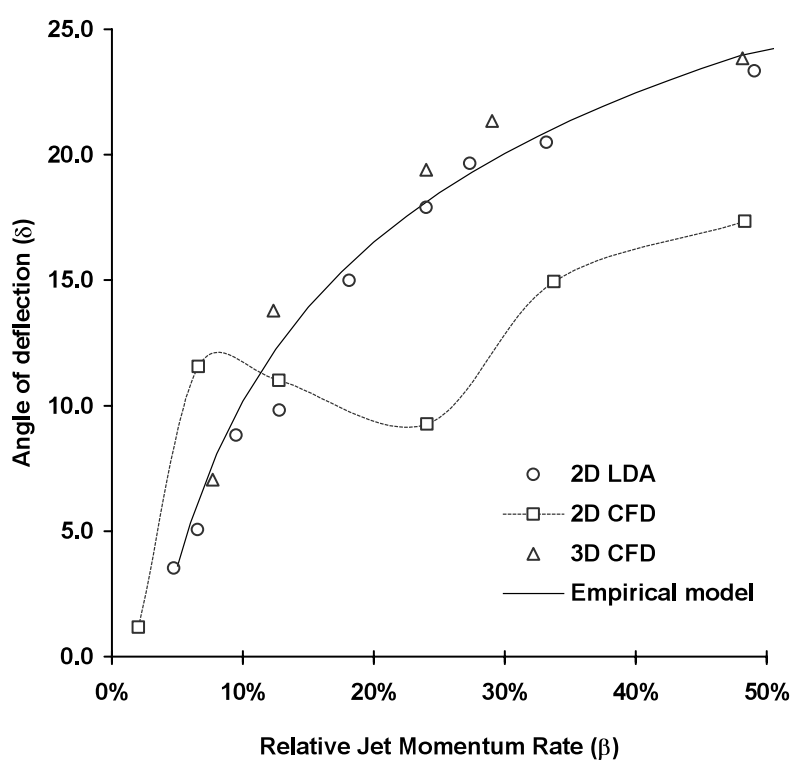

Fig. 7 LDA and CFD jet deflection characteristics $\left(Y_{1} / W=\right.$ 0.12 )
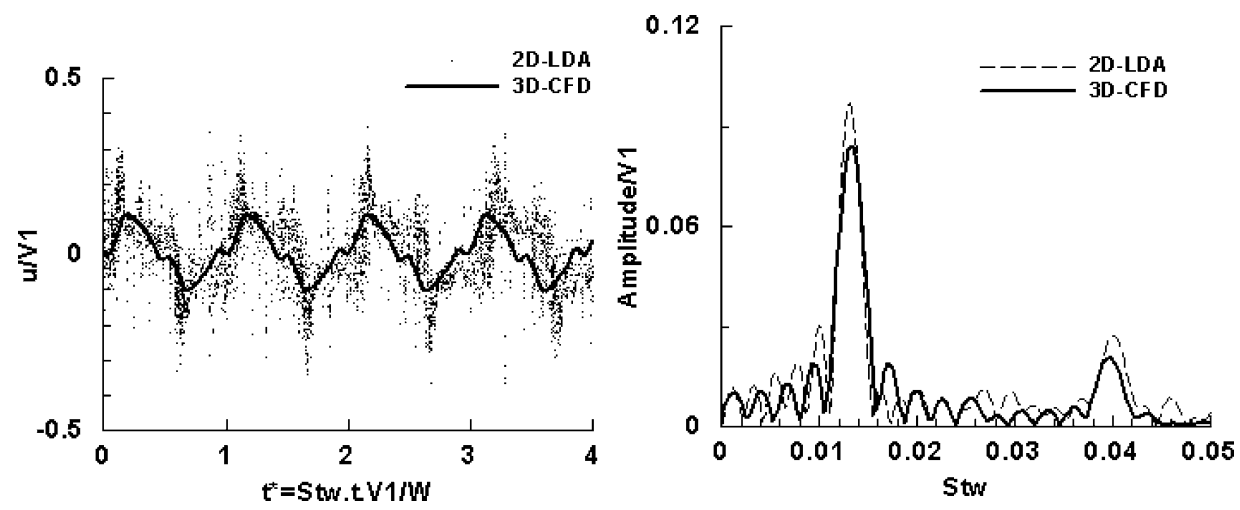

(a)
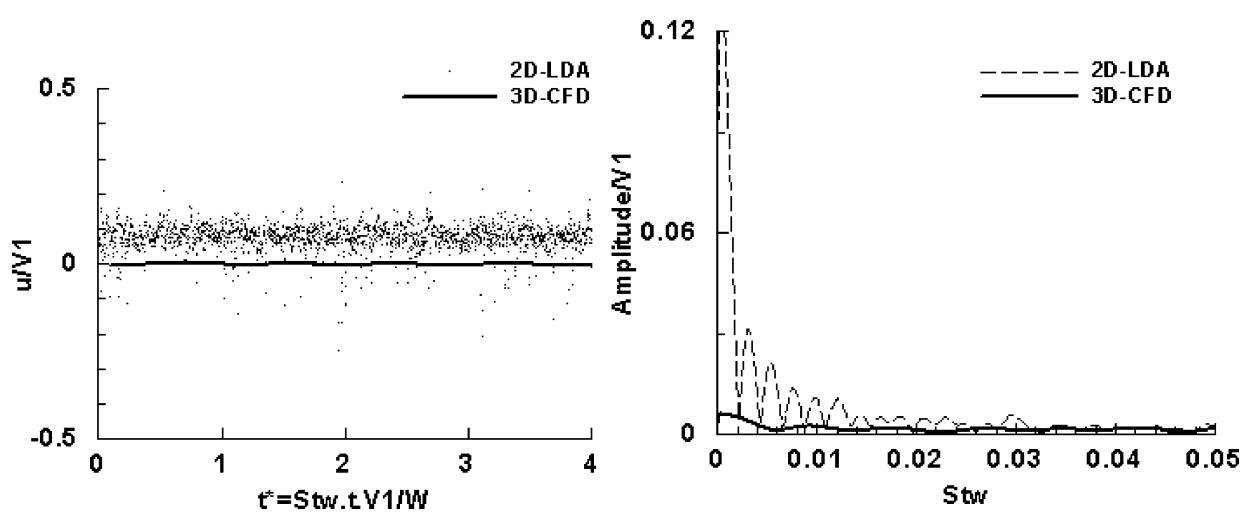

(b)

Fig. 8 LDA and 3D CFD spectral characteristics of the jet $\left(V_{1}=3.75 \mathrm{~m} / \mathrm{s}, Y_{1} / W=0.12\right)(\mathrm{a})$ $\beta=0$ (b) $\beta=49 \%$ 
responding to a Strouhal number of $S t_{W} \approx 0.013$. This characteristic has been previously reported by Lawson and Davidson $^{(8)}$ and was also captured in this case by the 3D CFD model. With secondary injection present, however, mean jet deflection was observed and the oscillation became less stable. In this case, the LDA cross flow data contained bursts of higher cross flow velocities at irregular intervals although the 3D oscillation appeared heavily damped. At the highest value of $\beta=49 \%$ shown in Fig. 8 (b), the primary jet was still found to move transversely across the cavity. However, the cross-flow amplitude, which is thought to be representative of jet movement, was greatly reduced with less unsteadiness. This trend was also captured by the 3D CFD although the major turbulent fluctuations were not observed.

\section{Conclusions}

This work has presented a method of flow control for a low Strouhal number $\left(S_{t}<0.02\right)$, self-sustained oscillating jet confined in a thin rectangular cavity. Flow control was achieved by secondary injection from a cavity sidewall jet. An LDA study using a water model and a CFD study using Fluent was used to investigate the performance of the system in different configurations over a range of secondary injection momentums. Initial results from the experimental system indicated greatest flow control sensitivity with injection above the nozzle exit into the crossflow region. This characteristic was also found using the 3D CFD model although the 2D CFD was not found to capture these properties accurately due to the 3D nature of the cross-flow region. Transient characteristics, however, were not faithfully captured by either the $2 \mathrm{D}$ or $3 \mathrm{D}$ CFD models although the 3D model predicted the general trends including changes in cross flow rms velocity. Future work aims to optimise the flow control system further by minimising cross flow below the nozzle exit and investigating the use of a synthetic jet secondary source. Further studies are also planned to improve the transient characteristics of the 3D CFD model.

\section{References}

( 1 ) Kral, L.D., Active Flow Control Technology, ASME Fluids Engineering Division Technical Brief, (1999).

( 2 ) Gad-el-Hak, M., Flow Control: Passive, Active, and
Reactive Flow Management, (2000), Cambridge University Press.

( 3 ) Alvi, F.S., Strykowski, P.J., Krothapalli, A. and Forliti, D.J., Vectoring Thrust in Multiaxes Using Confined Shear Layers, Journal of Fluids Engineering, Vol.122 (2000), pp.3-13.

( 4 ) Schmid, G.F., Strykowski, P.J., Madruga, M., Das, D. and Krothapalli, A., Jet Attachment Behavior Using Counterflow Thrust Vectoring, Proceedings of 13th ONR Propulsion Conference, Minneapolis, MN, Aug., 10-12, (2000), pp.63-68.

( 5 ) Smith, B.L. and Glezer, A., Vectoring and Small Scale Motions Effect in Free Shear Flows Using Synthetic Jet Actuators, 35th AIAA Aerospace Sciences Meeting, Reno, NV, AIAA Paper 97-0213 (unpublished), (1997).

( 6 ) Rockwell, D., Oscillations of Impinging Shear Layers, AIAA Journal, Vol.21 (1983), pp.645-664.

( 7 ) Lawson, N.J. and Davidson, M.R., An Investigation of a Low Strouhal Number Oscillatory Jet Submerged in a Thin Rectangular Cavity, 10th International Symposium on Application of Laser Techniques on Fluid Mechanics, Lisbon, Portugal, Paper 11.2., (2000).

( 8 ) Lawson, N.J. and Davidson, M.R., Self Sustained Oscillation of a Submerged Jet in a Thin Rectangular Cavity, Journal of Fluids and Structures, Vol.15 (2001), pp.59-81.

(9) Shakouchi, T., Suematsu, Y. and Ito, T., A Study on Oscillatory Jet in a Cavity, Bulletin of the JSME, Vol.25, No.206 (1982), pp.1258-1265.

(10) Shakouchi, T., Kuzuhara, S. and Yamaguchi, J., Oscillatory Phenomena of an Attached Jet, Bulletin of the JSME, Vol.29, No.250 (1986), pp.1117-1123.

(11) Gebert, B.M., Davidson, M.R. and Rudman M.J., Computed Oscillations of a Confined Submerged Liquid Jet, Applied Mathematical Modelling, Vol.22 (1998), pp.843-850.

(12) Lawson, N.J. and Davidson, M.R., Crossflow Characteristics of an Oscillating Jet in a Thin Slab Caster, Journal of Fluids Engineering, Vol.121 (1999), pp.589595.

(13) Arruda, M.P. and Lawson, N.J., Vectoring a SelfSustained Oscillatory Confined Jet Flow by Secondary Cross-Flow Injection, 42nd AIAA Aerospace Sciences Meeting and Exhibit, Reno, Nevada, 5th-8th January 2004, AIAA Paper 2004-0920, (2004).

(14) Arruda, M.P., Fluidic Vector Control of Self-Sustained Oscillatory Cavity Jets, Ph.D. Thesis, Cranfield University, (2004). 